\title{
USO DE OBSIDIANAS ENTRE LOS CAZADORES-RECOLECTORES EN EL BOSQUE DEL LAGO TRAFUL, NOROESTE DE LA PATAGONIA, ARGENTINA
}

\author{
LISANDRO G. LÓPEZ", MARIO J. SILVEIRA** Y CHARLES R. STERN"***
}

\begin{abstract}
RESUMEN
Se presentan los resultados de análisis geoquímicos realizados a artefactos de obsidiana procedentes de los sitios arqueológicos Alero Los Cipreses y Alero Lariviere, ubicados en el área del lago Traful, sur del Neuquén, Argentina. La obsidiana fue utilizada en forma continua en el área por grupos de cazadoresrecolectores durante los últimos 3.500 años. Hasta el momento se desconocía la cantidad y localización de las fuentes de aprovisionamiento utilizadas. Los resultados obtenidos es este estudio indican el uso de dos tipos de obsidiana predominantes sin interrupción y sugieren probables rangos regionales de movilidad e intercambios de esta materia prima.
\end{abstract}

PALABRAS CLAVES: artefactos de obsidiana, fuentes de obsidiana, tipos químicos de obsidiana, Neuquén, Patagonia, Argentina.

\section{OBSIDIAN USE AMONG HUNTER-GATHERERS IN THE TRAFUL LAKE WOODLAND, NORTHWEST PATAGONIA, ARGENTINA}

\section{ABSTRACT}

The results of a geochemical analysis of obsidian artifacts from the archaeological sites Los Cipreses and Lariviere rock-shelter, located in the area of Traful lake, southern Neuquén, Argentina are presented. Obsidian was utilized in this area continuously over the last 3.500 years by groups of hunter-gatherers. Until now, the number and location of the sources where this obsidian was obtained was unknown. The results obtained in this study indicate the use of two specific types of obsidian without interruption and constrain probable regional range of movement and interchange of this raw material.

KEYWORDS: obsidian artifacts, obsidian sources, obsidian chemistry, Neuquén, Patagonia, Argentina.

* Lisandro G. López. Centro de Arqueología Urbana (FADU-UBA). Ciudad Universitaria, pabellón 3, 4ํㅗㅇ. Ciudad Autónoma de Buenos Aires, CP 1636. E-mail: lisandroglopez@yahoo.com.ar

* Mario J. Silveira. Centro de Arqueología Urbana (FADU-UBA). Ciudad Universitaria, pabellón 3, 4º piso. Ciudad Autónoma de Buenos Aires, CP 1636. E-mail silveira@arnet.com.ar

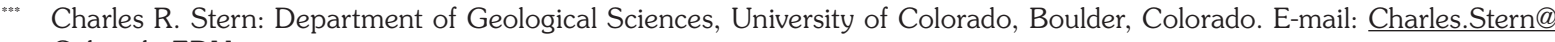
Colorado.EDU 


\section{INTRODUCCIÓN}

Las primeras ocupaciones en la zona de bosque lacustre cordillerano, en la costa norte del lago Traful, están datadas aproximadamente en 3500 años AP (Silveira 2003). La tecnología lítica incluye el uso de distintas clases de materias primas, entre ellas la obsidiana. En este trabajo se presentan los resultados de análisis geoquímicos realizados para determinar la procedencia de artefactos de obsidiana de los sitios arqueológicos Alero Los Cipreses (ALC), ubicado en la margen norte y media del lago Traful, y Alero Lariviere (AL), ubicado en las nacientes del arroyo Córdoba, uno de los afluentes principales del río Traful (Fig. 1) (Silveira 1988-1989, 1996, 1999). En esos sitios las obsidianas fueron utilizadas en forma continua desde las primeras ocupaciones hasta momentos post-hispánicos, sin conocerse la ubicación exacta de las fuentes de aprovisionamiento (Silveira 2003). Recientemente, se localizaron fuentes de obsidiana en dos áreas, en los lagos Lolog y Lácar, y también fueron reconocidos otros tipos químicos de fuentes desconocidas (Pérez y López 2009; López el al. 2009a). En este contexto, los objetivos planteados en este trabajo fueron: 1) deter- minar las fuentes de aprovisionamiento utilizadas en el lago Traful, y 2) si existieron diferencias a través del tiempo en su explotación.

\section{DESCRIPCIÓN DE LOS SITIOS}

\section{Alero Los Cipreses (ALC)}

El ALC está ubicado en la margen norte del lago Traful (40³9'47"S y 71ํ19'5”W), a 20 m sobre el nivel del lago y a unos $850 \mathrm{msnm}$ (Silveira 1996). Se encuentra al pie de un farallón rocoso que presenta arte rupestre y está rodeado por un bosque de cipreses (Austrocedrus chilensis). Las excavaciones se realizaron durante los veranos de 1982 y 1983. Los restos culturales fueron ordenados según las unidades de excavación, sobre la base de indicadores culturales y básicamente niveles estratigráficos. El sitio fue dividido en tres componentes, dos con presencia de cerámica y uno pre-cerámico, que se presentan a continuación: 1) Componente Cerámico-Los Cipreses II, final (CC-LC II): no posee fechados, sin embargo la presencia de materiales atribuidos al momento post-hispánico, como fauna exótica (caballo) y cuentas de vidrio, lo ubicaría en una fecha posterior al siglo XVI; 2) Componente

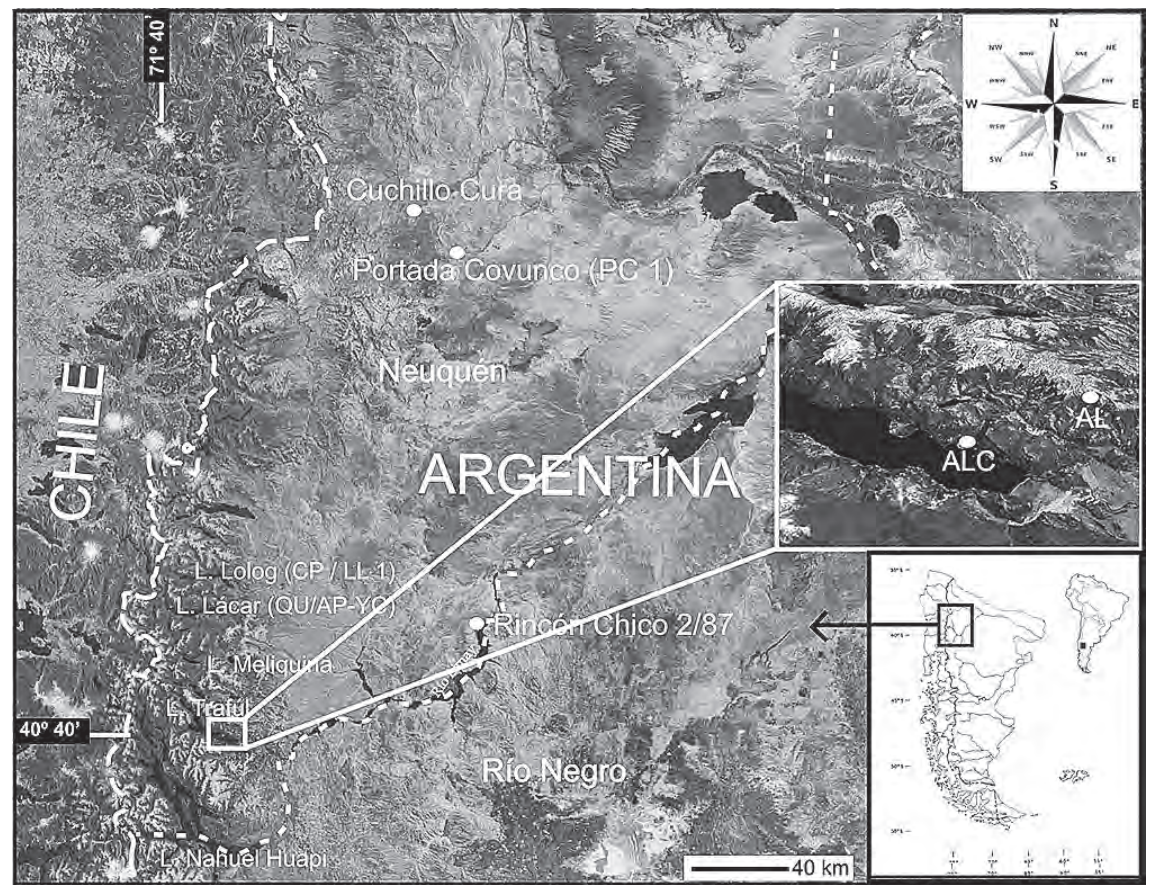

Fig. 1. Imagen satelital con la ubicación geográfica de fuentes de aprovisionamientos de obsidiana y sitiosáreas arqueológicas con información sobre análisis químicos de muestras geológicas y artefactos. 
Cerámico-Los Cipreses I (CC-LC I): datado por

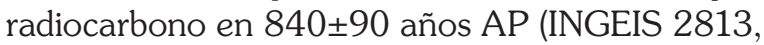
№ 954) ${ }^{1}$ y $1.510 \pm 90$ años AP (LATYR LP 145); y 3) Componente Acerámico-Los Cipreses (CA-LC): con fechados de $2.890 \pm 100$ años AP (INGEIS 2814, № 936) y de 3.490 \pm 80 años AP (LATYR LP 159) para la parte media, lo cual sugiere que las primeras ocupaciones del sitio serían algo anteriores a la última fecha. El ALC fue definido como un campamento residencial, donde quedaron registradas diferentes actividades representadas por el descarte de artefactos líticos, de hueso, asta, cerámica, pigmentos, distintas clases de adornos y restos de alimentos que evidencia el consumo de fauna muy variada (Silveira 1996).

\section{Alero Lariviere (AL)}

El AL está ubicado en las nacientes del arroyo Córdoba, uno de los afluentes principales del río Traful (40을 36 S y 71 13' W), a unos pocos metros de su margen derecha, en el límite entre los parques nacionales Nahuel Huapi y Lanín (Silveira 1988-1989, 1999). Está situado a 300 m sobre el nivel del lago Traful, a una distancia de $8 \mathrm{~km}$ de su costa norte y a $1300 \mathrm{msnm}$, en un paraje de bosque de coihue (Nothofagus donbeyi). El sitio fue prospectado en el verano de 1985, momento en el cual fue relevado el arte rupestre, y luego excavado en dos campañas durante los años 1986 y 1989. Posee niveles sin cerámica fechados en $2.760 \pm 80$ años AP (Beta-57836) y niveles con cerámica fechados en 780 \pm 50 años AP (Beta-57837). Debido a las frecuentes nevadas que dificultan los asentamientos en la zona durante una parte del año, el sitio fue definido como un campamento estacional o de "veranada" (Silveira 1999). El conjunto está compuesto casi exclusivamente por artefactos líticos, seguidos por los de cerámica, mientras que el instrumental óseo, adornos y otros objetos son muy escasos.

\section{FUENTES DE OBSIDIANA Y TIPOS QUÍMICOS CONOCIDOS}

Las fuentes conocidas de obsidiana más cercanas a los sitios estudiados están localizadas al norte del lago Traful (Fig. 1), donde fueron encontrados depósitos primarios y secundarias en

1 Todas las dataciones están sin calibrar. las cercanías de los lagos Lolog y Lácar (López et al. 2009a). En trabajos anteriores se evaluaron las características de cada fuente-cantidad, densidad, calidad y tamaño de los nódulos- siguiendo los criterios utilizados por Nami (1992), y se tomaron muestras para análisis geoquímicos (López et al. 2009a y 2009c).

Las obsidianas del lago Lácar están asociadas a dos tipos químicos diferentes denominados QU/ AP -Quilahuinto/Arroyo Pocahullo- y YC -Yuco. Los rodados de obsidianas QU/AP se hallaron en distintos sectores de la costa norte de ese lago, en las fuentes denominadas Turquesa, Playa Blanca y Arroyo Pocahullo, y en un sector del arroyo Quilahuinto, $3 \mathrm{~km}$ al norte de la costa del lago Lácar (López et al. 2009a, 2009c). En total se analizaron químicamente 20 muestras de rodados repartidos entre esas cuatro fuentes obteniendo resultados semejantes. La cantidad, densidad y el tamaño de los nódulos son mayores en el arroyo Quilahuinto en comparación con los depósitos de la costa del lago, aunque en general no superan los $5 \mathrm{~cm}$ de largo. En general, en ambas fuentes la distribución es aislada -una o dos rocas por $\mathrm{m}^{2}$ o menos de esa cantidad-y dispersa -dos a 10 rocas por $\mathrm{m}^{2}$. Si bien aún no se realizaron investigaciones al respecto, se sugiere el transporte natural de los nódulos desde una fuente primaria, actualmente desconocida, y probablemente localizada al norte del lago. En general, son obsidianas de color negro translúcido con bandas finas color gris, de excelente calidad para la talla y libre de cristales.

La obsidiana YC está asociada a una sola fuente secundaria compuesta por rodados de color negro translúcido depositados en una playa cercana a la localidad de Yuco, en la costa centro-norte del lago Lácar (López et al. 2009a). Se analizaron 14 muestras con resultados semejantes. Por lo general, los nódulos no superan los $5 \mathrm{~cm}$ de largo y se distribuyen en forma aislada y dispersa. Una particularidad de esta fuente es la presencia en gran parte de los nódulos de inclusiones de cristales de cuarzo de hasta $5 \mathrm{~mm}$ de largo, lo cual pudo haber afectado su calidad para la talla. De hecho, esta obsidiana no coincide químicamente con ninguna de las muestras de artefactos culturales analizados. Otro factor pudo ser la accesibilidad, debido a que la pendiente que desciende hacia la playa es muy pronunciada y con abundante vegetación. A pesar 
de esto, en la misma playa se hallaron unos pocos tiestos de cerámica, sin encontrarse artefactos líticos que indiquen actividades asociadas a la talla de esos rodados.

Cerca de la costa norte del lago Lolog hay un afloramiento primaria de obsidiana en el Cerro de las Planicies y depósitos secundarias de rodados dispersos en densidades variables en distintos sectores de la costa norte del lago, en Puerto Arturo y Costa Norte (López et al. 2009a). Las obsidianas del Cerro de las Planicies consisten en rodados y en menor medida clastos angulosos depositados en la cima de ese cerro, a una altura de 1700 msnm. Alli los nódulos son abundantes, se hallan concentrados -más de 10 rocas por $\mathrm{m}^{2}-\mathrm{y}$ se presentan en distintos tamaños. Se observaron y recolectaron varias muestras de entre 20 y $30 \mathrm{~cm}$ de diámetro. Además, se encontraron nódulos sin corteza y con evidencia de lascados, lo que indica algún trabajo en la cantera previo a su transporte. Por su parte, los rodados de la costa del lago se presentan generalmente aislados y dispersos, observándose mayor cantidad y tamaños más grandes en zonas más cercanas al Cerro de las Planicies como Puerto Arturo, localizado a $3,5 \mathrm{~km}$, aunque rara vez superan los $5 \mathrm{~cm}$ de diámetro. En sectores más alejados como Costa Norte, a $10 \mathrm{~km}$, no superan los $3,5 \mathrm{~cm}$. Esto indica el transporte natural hacia la costa del lago desde la cima del cerro. En general, los nódulos son muy homogéneos y de excelente calidad para la talla. Están representados distintos colores, tales como negro, negro con bandas grises y negro con bandas y/o manchas marrones o rojizas, de los cuales se extraen lascas, que según el espesor, pueden ser translúcidas a transparentes con bandas o manchas negras, o totalmente transparentes cuando son más delgadas. Los resultados de los análisis químicos realizados sobre muestras geológicas procedentes de esas fuentes permitieron distinguir un tipo químico predominante denominado CP/LL1 -Cerro de las Planicies/Lago Lolog 1- $(n=31)$ y uno menos frecuente, hallado hasta el momento solamente en Puerto Arturo, llamado CP/LL2 (n=2) (Pérez y López 2009; López et al. 2009a).

Además, Silveira (1996) reportó el hallazgo de pequeños rodados de obsidiana de color negro translúcido a transparente en una brecha localizada a unos $50 \mathrm{~m}$ del AL, donde aparecen incrustados en la roca en una formación de toba (Silveira 1996).
Es importante destacar que esos pequeños rodados aparecen en superficie y en estratigrafía tanto en AL, donde son más abundantes, como en ALC, y su diámetro es de entre 2,5 y $3 \mathrm{~cm}$. La presencia de lascas y puntas de proyectil muy pequeñas de obsidiana en ambos sitios sugiere que pudieron haber sido aprovechados para hacer instrumentos.

Otras fuentes importantes a destacar para los propósitos de este trabajo son las halladas en el centro del Neuquén. Se trata de fuentes secundarias de rodados de obsidiana asociadas al arroyo Covunco (Bellelli et al. 2006) y al Cerro Bayo (Stern et al. 2009). Ambas están vinculadas a dos tipos químicos bastante semejantes denominados PC1 -Portada Covunco 1- y CB -Cerro Bayo. Además, en esta misma área aparece un tipo químico menos frecuente denominado PC2 en la zona de Cuchillo Cura, el cual está asociado a artefactos y rodados de PC1 en lo que podría ser otra fuente secundaria (López et al. 2009a). Los rodados hallados en Cuchillo Cura son generalmente pequeños, inferiores a 4 $\mathrm{cm}$ de largo, de color negro translúcido y en menor medida negro con manchas marrones o rojizas y negro opaco. Si bien no existen estudios detallados al respecto, los artefactos de obsidiana hallados en sitios arqueológicos cercanos a esas fuentes (por ej. Alero Tromen IV y cueva Chenque Haichol) sugieren la disponibilidad de nódulos de mayores tamaños y de muy buena calidad (Perrotta y Pereda 1987; Fernández 1991).

Por último, los análisis realizados sobre artefactos de obsidiana procedentes de los alrededores de los lagos Meliquina y Lácar demostraron que existen otros dos tipos químicos de fuentes desconocidas utilizados frecuentemente, denominados provisoriamente "Desconocida 1" y "Desconocida 2" (Pérez y López 2009; López et al. 2009a, López et al. 2009c). La obsidiana "Desconocida 1" es color negra, gris, negra con bandas color gris y, en menor medida, negra translúcida, mientras que "Desconocida 2" es generalmente negra translúcida. Ambas son de muy buena calidad para la talla.

\section{MATERIALES Y MÉTODOS ANALITÍCOS}

Fueron analizadas un total de 26 muestras del ALC y 10 del AL, seleccionadas con el objetivo de representar la mayor variabilidad macroscópica posible. Del ALC se analizaron: 12 artefactos y un 
pequeño rodado natural del CA-LC, un pequeño rodado y 10 artefactos del CC-LC I, y dos artefactos del CC-LC II. Del AL fueron analizados dos pequeños rodados hallados en superficie, cinco artefactos $y$ dos rodados de los niveles con cerámica, y un artefacto de obsidiana azul procedente de los niveles sin cerámica. Los resultados fueron comparados con la información disponible sobre muestras geológicas procedentes de las distintas fuentes de obsidiana y de artefactos de los tipos químicos previamente citados cuyas fuentes permanecen desconocidas. Los análisis se realizaron en la Universidad de Colorado (Boulder, EEUU), donde las muestras de obsidiana fueron molidas a un polvo muy fino el cual fue disuelto en ácido fluorhídrico. Una vez en solución, las muestras fueron analizadas por medio de ICP-MS (espectrometría de masa por plasma iónicamente acoplado) para obtener la composición de elementos traza en partes por millón (ppm). Los análisis de los estándares internos sugieren un error de $\pm 10 \%$ a los niveles de concentración presentados por los diferentes tipos de obsidianas.

\section{RESULTADOS}

En total se determinaron cinco tipos químicos diferentes, dos de los cuales ya habían sido registrados en otras áreas cercanas, en los lagos Meliquina y Lácar. Las dos obsidianas más utilizadas en ambos sitio (ALC y AL) corresponden a los tipos químicos

Tabla 1. Promedio y desvío estándar (elementos trazas en ppm) para los cinco tipos químicos de obsidiana hallados en ALC y AL. *Nódulos-rodados.

\begin{tabular}{|c|c|c|c|c|c|c|c|c|}
\hline \multirow{3}{*}{$\begin{array}{c}\text { Tipos } \\
\text { № }\end{array}$} & \multirow{2}{*}{\multicolumn{2}{|c|}{$\frac{\text { CP/LL } 1}{n=11}$}} & \multirow{2}{*}{\multicolumn{2}{|c|}{$\begin{array}{l}\text { Des } 1 \\
\mathrm{n}=18\end{array}$}} & \multirow{2}{*}{\multicolumn{2}{|c|}{$\begin{array}{l}\text { TR } 1 \\
n=5^{*}\end{array}$}} & \multirow{3}{*}{$\begin{array}{c}\text { Des 4 } \\
\mathrm{n}=1^{*} \\
-\end{array}$} & \multirow{3}{*}{$\begin{array}{c}\mathrm{OA} \\
\mathrm{n}=1 \\
-\end{array}$} \\
\hline & & & & & & & & \\
\hline & Media & $\mathrm{DE}$ & Media & $\mathrm{DE}$ & Media & $\mathrm{DE}$ & & \\
\hline $\mathrm{Cu}$ & - & - & - & - & - & - & - & 64 \\
\hline $\mathrm{V}$ & - & - & - & - & - & - & - & 373 \\
\hline $\mathrm{Cr}$ & - & - & - & - & - & - & - & 70 \\
\hline $\mathbf{N i}$ & - & - & - & - & - & - & - & 69 \\
\hline $\mathrm{Ti}$ & 871 & 72 & 1655 & 174 & 720 & 33 & 1207 & 15285 \\
\hline Mn & 876 & 47 & 618 & 22,7 & 323 & 13 & 607 & 999 \\
\hline Cs & 5,2 & 0,38 & 6,1 & 0,29 & 8,5 & 0,5 & 5,3 & 0,5 \\
\hline $\mathbf{R b}$ & 142 & 4,7 & 157 & 5,1 & 210 & 2,9 & 112 & 30 \\
\hline $\mathrm{Sr}$ & 41,3 & 2,8 & 80 & 3,2 & 19,4 & 2,5 & 166 & 429 \\
\hline $\mathbf{B a}$ & 700 & 61 & 493 & 23 & 77,8 & 16,8 & 743 & 391 \\
\hline $\mathbf{Y}$ & 20,7 & 1,2 & 27 & 1 & 20 & 0,7 & 11 & 30 \\
\hline $\mathrm{Zr}$ & 93,6 & 3,9 & 285 & 8,2 & 92,8 & 4,65 & 106 & 210 \\
\hline $\mathbf{N b}$ & 16,8 & 0,6 & 21,3 & 0,6 & 22 & 0,7 & 8 & 18 \\
\hline Th & 11 & 0,8 & 18,2 & 1 & 24,6 & 6,8 & 14,8 & 2,2 \\
\hline Hf & 3,5 & 0,24 & 7,8 & 0,45 & 3,96 & 0,6 & 5,0 & 5,0 \\
\hline $\mathbf{P b}$ & 19,8 & 2,9 & 24 & 3,3 & 20,5 & 0,85 & 24,4 & 19,4 \\
\hline La & 12,5 & 1,36 & 36,5 & 1,6 & 30,3 & 2,6 & 22,88 & 18,6 \\
\hline Ce & 28 & 2,57 & 74,5 & 2,2 & 58 & 4,2 & 44,24 & 42,3 \\
\hline Pr & 3,14 & 0,27 & 7,8 & 0,3 & 5,8 & 0,4 & 4,35 & 5,65 \\
\hline Nd & 11,7 & 0,94 & 29,2 & 1,4 & 20,5 & 1,09 & 14,90 & 27,3 \\
\hline Sm & 3,1 & 0,24 & 5,3 & 0,2 & 3,4 & 0,2 & 2,74 & 7,02 \\
\hline Eu & 0,63 & 0,04 & 0,8 & 0,03 & 0,22 & 0,03 & 0,65 & 2,14 \\
\hline Gd & 3,43 & 0,24 & 5,8 & 0,23 & 4 & 0,16 & 2,88 & 8,06 \\
\hline Tb & 0,53 & 0,05 & 0,77 & 0,03 & 0,52 & 0,03 & 0,32 & 1,09 \\
\hline Dy & 3,47 & 0,19 & 4,6 & 0,22 & 3,24 & 0,09 & 1,97 & 6,32 \\
\hline Ho & 0,69 & 0,05 & 0,95 & 0,05 & 0,67 & 0,05 & 0,36 & 1,14 \\
\hline Er & 2,19 & 0,16 & 3,06 & 0,11 & 2,07 & 0,11 & 1,16 & 3,16 \\
\hline Tm & 0,32 & 0,03 & 0,47 & 0,02 & 0,32 & 0,02 & 0,21 & 0,40 \\
\hline $\mathbf{Y b}$ & 2,27 & 0,17 & 3,3 & 0,14 & 2,3 & 0,13 & 1,38 & 2,59 \\
\hline Lu & 0,33 & 0,03 & 0,5 & 0,03 & 0,33 & 0,02 & 0,21 & 0,35 \\
\hline
\end{tabular}


Tabla 2: Tipos químicos de obsidianas para las muestras del ALC y el AL. * Nódulo-rodado.

\begin{tabular}{|c|c|c|c|}
\hline \multicolumn{4}{|c|}{ ALERO LOS CIPRESES (ALC) } \\
\hline \multicolumn{2}{|c|}{ CA-LC $(2890 \pm 100$ y $3490 \pm 90$ años AP $)$} & \multicolumn{2}{|c|}{ CC-LC I $(840 \pm 90$ y $1510 \pm 90$ años AP) } \\
\hline ALC-1 & CL/LL 1 & ALC-14 & Desconocida 1 \\
\hline ALC-2 & CL/LL 1 & ALC-15 & Desconocida 1 \\
\hline ALC-3 & CL/LL 1 & ALC-16 & Desconocida 1 \\
\hline ALC-4 & Desconocida 1 & ALC-17 & CL/LL 1 \\
\hline ALC-5 & Desconocida 1 & ALC-18 & CL/LL 1 \\
\hline ALC-6 & CL/LL 1 & ALC-19 & Desconocida 1 \\
\hline ALC-7 & Desconocida 1 & ALC-21 & CL/LL 1 \\
\hline ALC-8 & Desconocida 1 & ALC-22 & Desconocida 1 \\
\hline ALC-9 & Desconocida 1 & ALC-23 & CL/LL 1 \\
\hline ALC-10 & CL/LL 1 & ALC-25 & Desconocida 1 \\
\hline ALC-11 & Desconocida 1 & ALC-26 & TR $1^{*}$ \\
\hline ALC-12 & Desconocida $4^{*}$ & \multicolumn{2}{|c|}{ CC-LC II (Posterior al siglo XVI) } \\
\hline ALC-13 & Desconocida 1 & ALC-20 & Desconocida 1 \\
\hline - & - & ALC-24 & Desconocida 1 \\
\hline \multicolumn{4}{|c|}{ ALERO LARIVIERE } \\
\hline \multicolumn{2}{|c|}{ NIVELES SIN CERAMICA $(2760 \pm 80$ años AP) } & AL-34 & Desconocida 1 \\
\hline AL-31 & Obsidiana Azul (OA) & AL-35 & CL/LL 1 \\
\hline \multicolumn{2}{|c|}{ NIVELES CON CERAMICA $(780 \pm 90$ años AP) } & AL-36 & CL/LL 1 \\
\hline AL-29 & TR $1^{*}$ & \multicolumn{2}{|c|}{ SUPERFICIE } \\
\hline AL-30 & TR $1^{*}$ & $\mathrm{Al}-27$ & TR $1^{*}$ \\
\hline AL-32 & Desconocida 1 & AL-28 & TR $1^{*}$ \\
\hline AL-33 & Desconocida 1 & - & - \\
\hline
\end{tabular}

CP/LL1 y "Desconocida 1". La primera proviene de las fuentes localizadas en la zona del lago Lolog, entre 70 y $75 \mathrm{~km}$ al norte del los sitios estudiados. La procedencia del tipo "Desconocida 1" permanece sin ser ubicada. En la Tabla 1 se presenta el promedio y desvío estándar por elemento para los distintos tipos químicos de obsidiana hallados en los dos sitios, mientras que en la Tabla 2 se pueden observar las fuentes correspondientes para cada una de las muestras analizadas. Por su parte, en la Fig. 2 se presenta la relación entre $\mathrm{Zr}$ vs $\mathrm{Ba}$ comparando las muestras del ALC y el AL con las obsidianas de las fuentes del lago Lolog (CP/LL1), del lago Lácar (QU/AP y YC), de Portada Covunco (PC1) y con los tipos químicos "Desconocida 1" y "Desconocida 2".

La obsidiana CP/LL1 procedente del lago Lolog está representada por dos artefactos de los niveles con cerámica del AL y por 9 del ALC. De estos últimos, cinco corresponden al CA-LC y cuatro al CC-LC I. Por su parte, la obsidiana denominada "Desconocida 1" está representada por tres artefactos de los niveles con cerámica del AL y por 15 artefactos del ALC, 7 de los cuales corresponden el CA-LC, 6 al CC-LC I y dos al CCLC II. Esta información indica que las obsidianas de ambas fuentes fueron utilizadas en el área en forma prácticamente continua desde aproximadamente 3.490 90 años AP hasta momentos históricos posteriores al siglo XVI.

La obsidiana azul oscura del AL (AL-31) es químicamente muy diferente a las otras obsidianas analizadas en la región. Presenta muy alta concentración de $\mathrm{Cu}$-usualmente por debajo del límite de detección- y relativamente alto $\mathrm{V}, \mathrm{Cr}$ y Ni (Tabla 3 ), lo que sugiere que esta muestra fue afectada por fluidos ricos en esos metales y que el color azul puede estar relacionado con la influencia de ellos, en particular el cobre. Además, comparada con las otras obsidianas conocidas en la región, tiene muy baja concentración de Cs y Th y alta de Sr y Ti, lo cual puede también reflejar la alteración química secundaria que produjo el color azul.

Los cuatro rodados de obsidiana analizados del AL y uno del CC-LC I del ALC (ALC-26) son químicamente semejantes. Su presencia coincide con la proximidad de la fuente -cercana al AL-, por lo que denominamos a este tipo TR1-Traful 1 . 


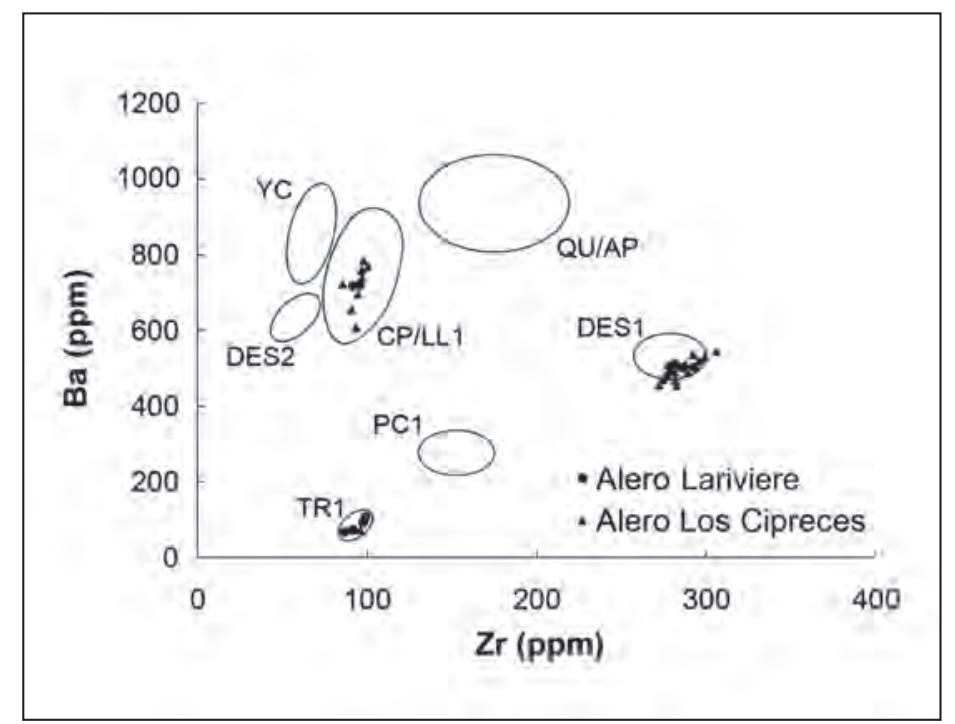

Fig. 2. Relación de Zr us Ba en ppm para muestras de los tres tipos químicos más representados en ALC y AL (CP/LL1, Des1 y TR1) en realación con las fuentes de obsidiana de Portada Covunco (PC1), lago Lolog (CP/LL1) y lago Lácar (QU/AP y YC) y los tipos químicos de fuentes desconocidas (Des1 y Des2).

El otro pequeño rodado del CA-LC (ALC-12) es diferente a los anteriores y a los demás tipos de obsidiana conocidos, por lo cual se lo denomina provisoriamente Desconocida 4. En ambos casos, su uso para hacer instrumentos no fue constatado entre los demás artefactos analizados.

\section{DISCUSIÓN Y CONCLUSIONES}

La obsidiana fue utilizada en los sitios estudiados en forma recurrente, principalmente para la manufactura de puntas bifaciales y ocasionalmente de lascas no retocados con rastros complementarios, instrumentos con microretoques -raclettes- , muescas y en menor medida perforadores, raspadores $y$ otros instrumentos unifaciales y bifaciales. Su presencia en el registro arqueológico representa, en parte, los rangos anuales de movilidad de los cazadores-recolectores que habitaron en el bosque en el área circundante al lago Traful. Los desechos de talla -lascas, microlascas y fragmentos de distintos tamaños- hallados en ambos sitios indican que se realizaron actividades de producción y mantenimiento de instrumentos. La ausencia de núcleos sugiere que esa materia prima ingresó mayoritariamente como formas bases, preformas e instrumentos terminados. Si bien, la presencia de algunos fragmentos indiferenciados -posiblemente núcleos residuales- $y$ artefactos que mantienen reserva de corteza indicaría que los núcleos también eran transportados, pero conservados y descartados una vez agotados por completo.

Los resultados presentados en este trabajo muestran que se utilizaron dos tipos de obsidiana principales: CP/LL1 y "Desconocida 1". Estas están presentes desde las primeras ocupaciones datadas en 3.500 años AP hasta el período post-hispánico, posterior al siglo XVI, lo cual representa cierta continuidad en su acceso. Además, fue utilizada una obsidiana color azul -OA- y transportados a los sitios pequeños rodados. La obsidiana azul pudo ser valorada principalmente por su color y escasez. Esta obsidiana no fue encontrada en ninguno de los otros sitios del área; sólo aparecen dos lascas en el los niveles sin cerámica 18 y 20 del AL, por debajo de los niveles 21 a 24 fechados en 2760 \pm 80 años AP. Artefactos de obsidiana del mismo color -3 lascas- fueron encontrados, también en niveles sin cerámica, en el sitio arqueológico El Trébol, ubicado en la margen sur del lago Nahuel Huapi (Hajduk et al. 2004). Los datos actuales sugieren que su uso habría sido poco frecuente y restringido a un determinado momento.

Con respecto a los pequeños rodados de obsidiana, Ceballos (1982) también reporta su presencia en distintos niveles de la cueva Cuyín Manzano, 
ubicada aproximadamente a $15 \mathrm{~km}$ al sudeste del lago Traful. La misma autora comenta que entre las colecciones pertenecientes a Parques Nacionales del Museo Perito Moreno de Bariloche, se encuentran piedras de obsidiana del mismo tipo a las que se halló en un pequeño trozo de cuero, que debería haber cumplido la función de una bolsita para que el cazador pudiera llevarla consigo; desconociéndose su lugar de origen (Ceballos 1982: 36). Sin embargo, los datos actuales sugieren que los pequeños rodados hallados en ALC y AL probablemente no fueran utilizados para hacer instrumentos, por lo que su presencia en sitios arqueológicos del sur neuquino es una incógnita.

Uno de los resultados menos esperados es la ausencia entre las muestras analizadas de la obsidiana QU/AP procedente del área del lago Lácar (Fig. 1), la fuente más cercana al lago Traful, localizada entre 50 y $55 \mathrm{~km}$ de los sitios estudiados. Esta fue utilizada en las áreas de los lagos Meliquina $(n=3)$ y Lácar $(n=3)$, donde fueron encontrados artefactos de esa materia prima (López et al. 2009a). La ausencia de YC, también procedente del lago Lácar, se debería a que presenta cristales de cuarzo de hasta $5 \mathrm{~mm}$ que habrían disminuido su utilidad, sobre todo dada la disponibilidad de otras obsidianas de mejor calidad. Tampoco fue encontrada la obsidiana denominada "Desconocida 2" hallada en varias muestras de artefactos analizados procedentes de los lagos $\mathrm{Me}$ liquina ( $n=10)$ y Lácar ( $n=3$ ) (Pérez y López 2009; López et al. 2009a, 2009c). Desconocida 1 fue hallada en Traful $(n=18)$ y en Meliquina $(n=13)$, pero no en el lago Lácar (Pérez y López 2009; López et al. 2009a). Por su parte, la obsidiana CP/LL1 procedente del lago Lolog (Fig. 1), localizada entre 70 y $75 \mathrm{~km}$ al norte del los sitios estudiados, es la única que aparece en las tres áreas -Traful ( $n=11)$, Meliquina ( $n=5)$ y Lácar ( $n=5)$, lo que sugiere el uso selectivo de la misma a pesar de la distancia a la fuente de aprovisionamiento. Es importante citar también que CP/LL1 fue hallada en sitios arqueológicos de la Pampa Seca, a $520 \mathrm{~km}$ al noreste de la fuente (Giesso et al. 2008; López et al. 2009b), y en la costa Atlántica de Río Negro, a 560 km al este de la fuente (Favier Dubois et al. 2009).

Por último, la obsidiana PC1, cuyas fuentes están localizadas en el centro del Neuquén (Bellelli et al. 2006; López et al. 2009a), hasta el momento no fue encontrada en el sudoeste neuquíno, en las zonas de Traful, Meliquina y Lácar. Sin embargo, un artefacto de obsidiana procedente del sitio arqueológico Rincón Chico 2/87, ubicado cerca de la ciudad de Piedra del Águila, aproximadamente a $150 \mathrm{~km}$ al sur de la fuente (Fig. 1), resultó ser químicamente semejante a PC1 (Palacios 2009), lo que indica que circulo hacia la zona de estepa del sur pero no hacia el sudoeste de la provincia, en la región de los lagos. Esta obsidiana fue hallada también en sitios arqueológicos del centro-sur de Chile, aproximadamente $70 \mathrm{~km}$ al oeste de la fuente (Stern et al. 2009), y en el área de Cholila -Chubut-, 430 $\mathrm{km}$ al sur (Bellelli et al. 2006).

En este contexto, los resultados de los análisis químicos realizados hasta el momento muestran cierta variación en la distribución regional de artefactos de distintos tipos de obsidiana, probablemente relacionada con la accesibilidad a las fuentes de aprovisionamiento, las características de las mismas -calidad, cantidad y tamaño de los nódulos-, con la movilidad humana y la existencia de distintas redes de intercambio de esa materia prima. Además, algunas obsidianas fueron elegidas para ser transportadas a mayor distancia (CP/LL1 y PC1), mientras que otras fueron utilizadas con mayor frecuencia en las cercanías de sus fuentes (QU/AP) o no fueron utilizadas para hacer instrumentos (YC).

\section{AGRADECIMIENTOS}

Agradecemos a todas las personas, estudiantes, graduados y colaboradores, que participaron de una u otra manera en este proyecto. Agradecemos también a Daniel Batres por su aporte y al Centro de Arqueología Urbana de la Universidad de Buenos Aires (FADU-UBA). Queremos dedicar este trabajo, en forma especial, a la memoria del muy estimado Sr. Mauricio Lariviere por su ayuda desinteresado. Los materiales analizados fueron obtenidos durante los trabajos que se realizaron en el marco del "Proyecto Arqueológico Traful" (PAT) que se desarrolló en la cuenca del lago Traful entre 1979 y 1995 en varias campañas, con apoyo de la Universidad de Buenos Aires, el Instituto de Ciencias Antropológicas de la Facultad de Filosofía y Letras, el Programa de Estudios Prehistóricos (PREP-CONICET) y la Administración General de Parques Nacionales. Finalmente, los autores asumimos la responsabilidad por lo presentado. 


\section{REFERENCIAS}

BELlELli, C., F. X. PEREYRA Y M. CARBALLIDO. 2006. Obsidian localization and circulation in northwestern Patagonia (Argentina): sources and archaeological record. En "Geomaterials in Cultural Heritage", editado por M. Maggetti y B. Messiga, pp. 241-255. Geological Society, Special Publications, London.

CEBALlOS, R. 1982. El Sitio Cuyín Manzano. Estudios y Documentos. Centro de Investigaciones Científicas de Río negro 9: 1-64.

FERNANDEZ, J. 1991. La cueva de Haichol. Arqueología de los pinares cordilleranos del Neuquén. Anales de Arqueología y Etnología 43/45, 1-3 (1988-1990): 1-740.

GIESSO, M., M. A. BERÓN Y M. D. GLASCOCK. 2008. Obsidian in Western Pampas, Argentina: Source Characterization and Provisioning Strategies. IAOS Bulletin 38: 15-38. Winter 2008.

HAJDUK, A., A: ALBORNOZ Y M.J. LEZCANO. 2004. El "MYLODON" En el Patio de Atrás. Informe Preliminar sobre los Trabajos en el Sitio El Trébol, Ejido Urbano de San Carlos de Bariloche. Provincia de Río Negro. Contra Viento y Marea. Arqueología de la Patagonia, compilado por M. T. Civalero, P. M. Fernández y A. G. Guraiéb, pp. 715-731. Instituto Nacional de Antropología y Pensamiento Latinoamericano-Sociedad Argentina de Antropología, Buenos Aires.

FAVIER DUBOIS, C; C. R. STERN Y M. CARDILLO. 2009. Primera caracterización de la distribución y tipos de obsidiana presentes en la costa rionegrina. En Arqueología de Patagonia: una mirada desde el último confín, editado por M. Salemme, F. Santiago, M. Álvarez, E. Piana, M. Vázquez y M.E. Mansur), Editorial Utopías (Ushuaia). En prensa.

LÓPEZ, L., A. PÉREZ Y C. R. STERN. 2009a. Fuentes de aprovisionamiento y distribución de obsidianas en la provincia de Neuquén, (Noroeste de la Patagonia Argentina). Intersecciones en Antropología 10: 75-88.

LÓPEZ, L., A. PÉREZ, D. BATRES Y C. R. STERN. 2009b. Obsidian Artifacts in La Pampa, Argentina, from Sources in Southwest Neuquén. IAOS Bulletin 41: 4-8.

LÓPEZ, L., A. PÉREZ Y C. R. STERN. 2009c. Descripción y caracterización química de distintas fuentes y tipos de obsidiana en la cordillera de Los Andes, sudoeste del Neuquén, Norpatagonia Argentina. Trabajo presentado en el XVIII Congreso de Nacional de Arqueología Chilena. Valparaíso.
NAMI, H. 1992. El subsistema tecnológico de la confección de instrumentos líticos y la explotación de los recursos del ambiente: una nueva vía de aproximación. Shincal 2: 33-53. Escuela de Arqueología. Universidad Nacional de Catamarca, Catamarca.

PALACIOS, O. M. 2009. Informe sobre dos muestras de obsidiana halladas en Rincón Chico 2/87. En Arqueología de rescate en Rincón Chico, provincia del Neuquén. Capítulo 11: 135-140. Copiladores. Eduardo A. Crivelli Montero, Mabel M. Fernández y Mariano S. Ramos. Editorial Dunken, Buenos Aires.

PÉREZ, A. Y L. LÓPEZ. 2009. Análisis por LA-ICP-MS para determinar las fuentes de aprovisionamiento de las obsidianas utilizadas en la Localidad Arqueológica Meliquina (Parque Nacional Lanín, Provincia de Neuquén). Actas de las I Jornadas Nacionales de Arqueología de Cazadores-Recolectores. Series Monográficas №2, Santiago. En prensa.

PERROTA, E. Y I. PEREDA. 1987. Nuevos datos sobre el Alero Tromen IV. (Dto. Picunches, Provincia del Neuquen). Actas de las I Jornadas de Arqueología de la Patagonia, pp. 249-258. Dirección Provincial de Cultura, Rawson.

SILVEIRA, M. J. 1988-1989. Un sitio con arte rupestre: El Alero Lariviere (Provincia del Neuquén). Relaciones de la Sociedad Argentina de Antropología. T.XVII/2: 75-86.

-1996. Alero Los Cipreses (provincia del Neuquén, República Argentina). Arqueología. Sólo Patagonia. Ponencias de las Segundas Jornadas de Arqueología de la Patagonia, editado por Julieta Gómez Otero, pp. 107-118. Centro Nacional Patagónico, Puerto Madryn. -1999. El Alero Lariviere: Un sitio en el bosque septentrional andino. (departamento de Los Lagos, provincia de Neuquén, Argentina). Soplando en el Viento... Actas de las Terceras Jornadas de Arqueología de la Patagonia, pp. 83-95. INAPL-Universidad del Comahue, Neuquén-Buenos Aires.

-2003. Las poblaciones prehistórica (s) e históricas en el área boscosa-ecotonal del lago Traful (provincia del Neuquén). III Congreso Argentino de Americanistas, Año 1999, III, pp. 399-418. Buenos Aires: Sociedad Argentina de Americanistas.

STERN, C. R., C. GARCIA, x. NAVARRO Y J. MUÑOZ. 2009. Fuentes y distribución de diferentes tipos de obsidiana en sitios arqueológicos del centro sur de Chile (38-44오). Magallania 37(1): 179-192. 
